This article was downloaded by: [National Taiwan University]

On: 25 March 2009

Access details: Access Details: [subscription number 908165551]

Publisher Taylor \& Francis

Informa Ltd Registered in England and Wales Registered Number: 1072954 Registered office: Mortimer House, 37-41 Mortimer Street, London W1T 3JH, UK

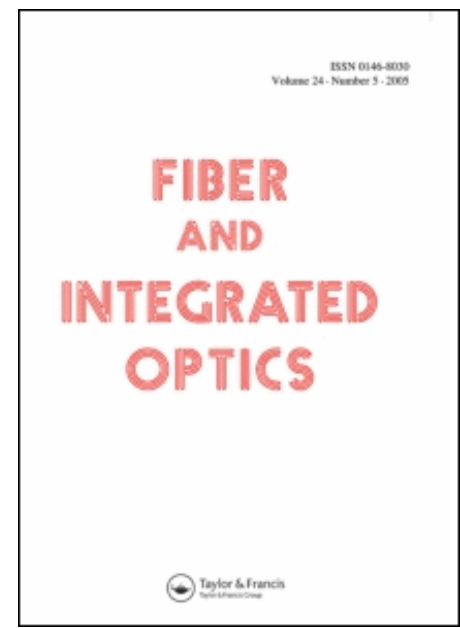

\title{
Fiber and Integrated Optics
}

Publication details, including instructions for authors and subscription information:

http://www.informaworld.com/smpp/title content=t713771194

\section{Polarization Control Technique to Reduce, Power Penalty in Tri-Directional,} Transmission Systems

Shien-Kuei Liaw a; Keang-Po Ho ${ }^{b}$

a Department of Electronic Engineering, National Taiwan University of Science and Technology, Taipei,

Taiwan, Republic of China. ${ }^{b}$ StrataLight Communications, Campbell, California, USA.

Online Publication Date: 01 July 2003

To cite this Article Liaw, Shien-Kuei and Ho, Keang-Po(2003)'Polarization Control Technique to Reduce, Power Penalty in Tri-

Directional, Transmission Systems',Fiber and Integrated Optics,22:4,275 - 282

To link to this Article: DOI: $10.1080 / 01468030390208466$

URL: http://dx.doi.org/10.1080/01468030390208466

\section{PLEASE SCROLL DOWN FOR ARTICLE}

\footnotetext{
Full terms and conditions of use: http://www.informaworld.com/terms-and-conditions-of-access.pdf

This article may be used for research, teaching and private study purposes. Any substantial or systematic reproduction, re-distribution, re-selling, loan or sub-licensing, systematic supply or distribution in any form to anyone is expressly forbidden.

The publisher does not give any warranty express or implied or make any representation that the contents will be complete or accurate or up to date. The accuracy of any instructions, formulae and drug doses should be independently verified with primary sources. The publisher shall not be liable for any loss, actions, claims, proceedings, demand or costs or damages whatsoever or howsoever caused arising directly or indirectly in connection with or arising out of the use of this material.
} 


\title{
Polarization Control Technique to Reduce Power Penalty in Tri-Directional Transmission Systems
}

\author{
SHIEN-KUEI LIAW \\ YI-FAN CHIANG
}

Department of Electronic Engineering

National Taiwan University of Science and Technology

Taipei, Taiwan, Republic of China

\author{
KEANG-PO HO \\ StrataLight Communications \\ Campbell, California, USA
}

\begin{abstract}
Polarization control is used in a tri-directional wavelength-reused system to reduce the power penalty caused by coherent mixing of the signal with Rayleigh backscattering noise. For a $25+25 \mathrm{~km}, 10 \mathrm{~Gb} / \mathrm{s}$ tri-directional transmission system, the power penalty is $4.0 \mathrm{~dB}$ under the worst polarization control and could be reduced to $0.4 \mathrm{~dB}$ under optimal polarization control. The experimental results show that reusing the same wavelength is an efficient and promising method for tri-directional lightwave systems.
\end{abstract}

Keywords wavelength division multiplexing, tri-directional transmission, wavelength reuse, Rayleigh backscattering, polarization control

\section{Introduction}

Wavelength-division-multiplexed (WDM)-based optical networks are indispensable for current and future high-capacity networking infrastructure. Compared with unidirectional transmission, bi-directional transmission over a single fiber reduces the required number of fibers and thus reduces the cost. Some of the new optical system architectures are proposed using only one fiber for the transmission in both directions [1-2]. As the demand on information capacity grows, tri-directional transmission will be another useful solution for network design, signals interchange, and cross-connecting purposes. Conventional systems use different wavelengths for counter-propagating WDM signals to avoid overlapping the spectra. Although the Rayleigh backscattering (RB) can be reduced [3-5], it will restrict the maximum spectral efficiency.

Received 11 July 2002; accepted 8 January 2003.

The work is partially supported by the National Science Council (NSC), R.O.C., under grant NSC R-091-00318 and NSC 91-2622-L-011-001.

Address correspondence to Shien-Kuei Liaw, National Taiwan University of Science and Technology, No. 43, Sec. 4, Keelung Rd., Taipei, Taiwan 106. E-mail: skliaw@et.ntust.edu.tw 
We report the usage of an identical wavelength for counter-propagating to increase the spectral efficiency in a bi-directional system [6]. If $\lambda$ and $\lambda^{\prime}$ are the counter-propagating signals with the same wavelength in the reverse direction, the signal coherently mixes with RB generated from the counter-propagating signals and thus induces homodyne crosstalk at the receiver. However, no methods are suggested to reduce the power penalty due to RB.

On the other hand, we found that homodyne crosstalk may induce a $1.5 \mathrm{~dB}$ power penalty under a $-20 \mathrm{~dB}$ interference signal [7], and ordinary homodyne crosstalk is equivalent to backward propagating light due to discrete reflection as compared with the distributed nature of RB. Thus, the penalty induced by RB may be one of the major causes of degradation in bi-directional and tri-directional WDM systems, especially when the identical wavelengths are used in each direction. In an optical network or lightwave system, a tri-directional system may be equivalent to several bi-directional systems. A tri-directional transmission may include wavelength cross-connect and/or wavelength add/drop functions, while it seldom appears in a bi-directional system. In this article we study the tri-directional transmission and use polarization control technology to reduce the power penalty.

\section{Rayleigh Backscattering Issues}

Polarization control between counter-propagating signals can reduce the impact of RBinduced homodyne crosstalk. It is based on the assumption that polarization mode dispersion (PMD) normally leads to the requirement of orthogonal polarization between counter-propagating signals. If the counter-propagating signals follow the two orthogonal principal states of polarization (PSP), PMD induces no distortion to each signal. In this article we propose to use the identical wavelength in a tri-directional transmission system. We study the possibility of controlling the polarization state of signals to reduce the power penalty by theoretic analysis and experimental demonstration.

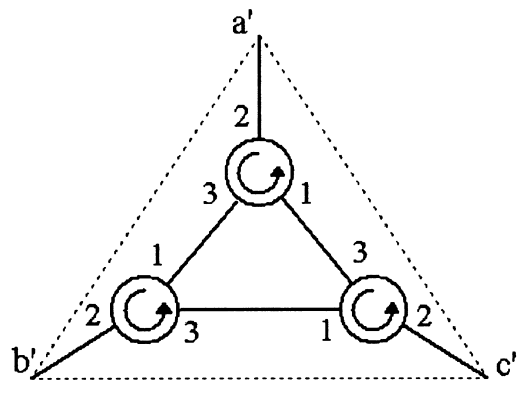

(a)

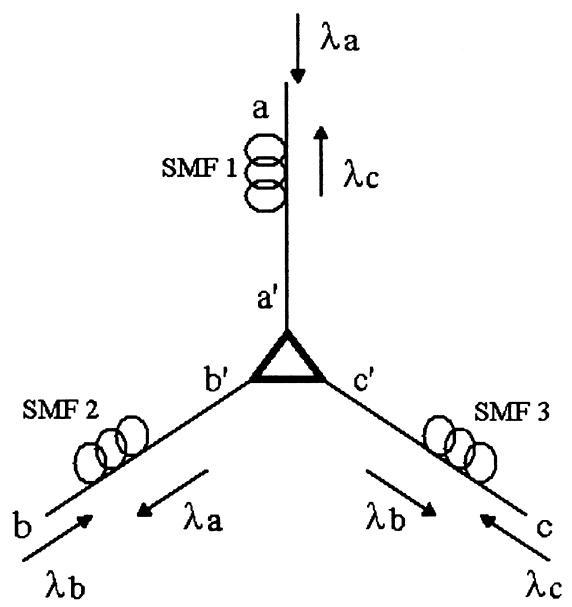

(b)

Figure 1. (a) Proposed tri-directional transmission module (TTM). (b) TTM in a tri-directional transmission system. 


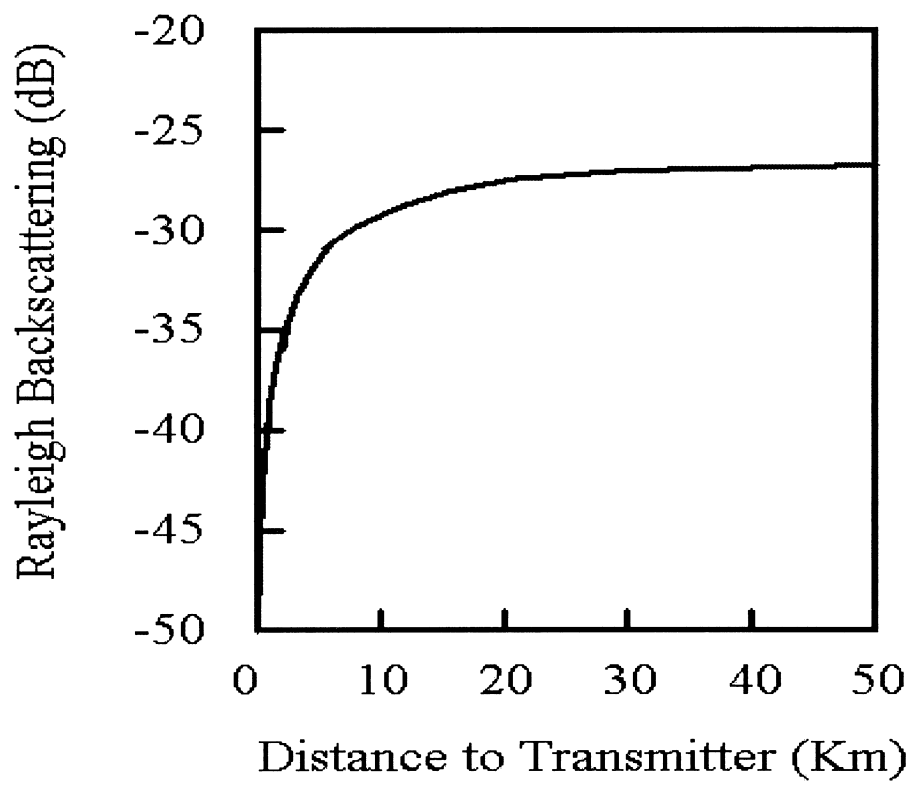

Figure 2. Simulated results of the RB effect against fiber transmission distance.

Figure 1a shows the proposed triangle box as a tri-directional transmission module (TTM). It consists of at least three pieces of Optical Circulators (OCs). Figure 1b shows the TTM in a tri-directional system where $\lambda_{a}$ travels from point a to point $\mathrm{b}, \lambda_{b}$ travels from point $\mathrm{b}$ to point $\mathrm{c}$, and $\lambda_{c}$ travels from point $\mathrm{c}$ to point $\mathrm{a}$. Usually tri-directional transmission is more complicated than bi-directional transmission. For example, in Figure $1 \mathrm{~b}$, when $\lambda_{a}$ travels from a to $\mathrm{b}$, the main interference signal from a to $\mathrm{a}^{\prime}$ is $\lambda_{c}$, while from $\mathrm{b}^{\prime}$ to $\mathrm{b}$ is $\lambda_{b}$. Figure 2 simulates the RB effect against the fiber transmission distance. The RB level varies with various fiber lengths without considering its polarization state. For the propagation of $\lambda_{a}$ from point a to point $\mathrm{b}, \lambda_{c}$ is the interference signal in SMF1, while $\lambda_{b}$ is the interference signal in SMF2. With the optical signals launching into a proposed TTM, the total amount of RB is randomly distributed, that is, it may be in any point of the Poincaré sphere. However, the statistical properties of RB polarization depend on the launched polarization. If the Stokes vector of the launched signal is $\left(\mathrm{s}_{1}, \mathrm{~s}_{2}, \mathrm{~s}_{3}\right)$, for far-away $\mathrm{RB}$, the total power in the direction parallel to $\left(\mathrm{s}_{1}\right.$, $\mathrm{s}_{2},-\mathrm{s}_{3}$ ) is two times more than that in the orthogonal direction [8]. If the launched signal is linearly polarized along the equator of the Poincare sphere with $\mathrm{s}_{3}=0, \mathrm{RB}$ is minimized in the orthogonal direction. Normally the laser output has linear polarization. Controlling the counter-propagating signals into orthogonal polarization can minimize homodyne crosstalk due to RB.

\section{Experimental Setup}

Figure 3 depicts the experimental setup to study a polarization-controlled, wavelengthreused tri-directional transmission. A $10 \mathrm{~Gb} / \mathrm{s}$ modulated signal with pseudo-random bit sequence (PRBS), $2^{31}-1$ non-return-to-zero (NRZ) data stream is used to modulate externally a distributed feedback (DFB) laser at $1546.0 \mathrm{~nm}$. After passing through an erbium-doped fiber amplifier (EDFA), a variable optical attenuator (VOA) is used to 


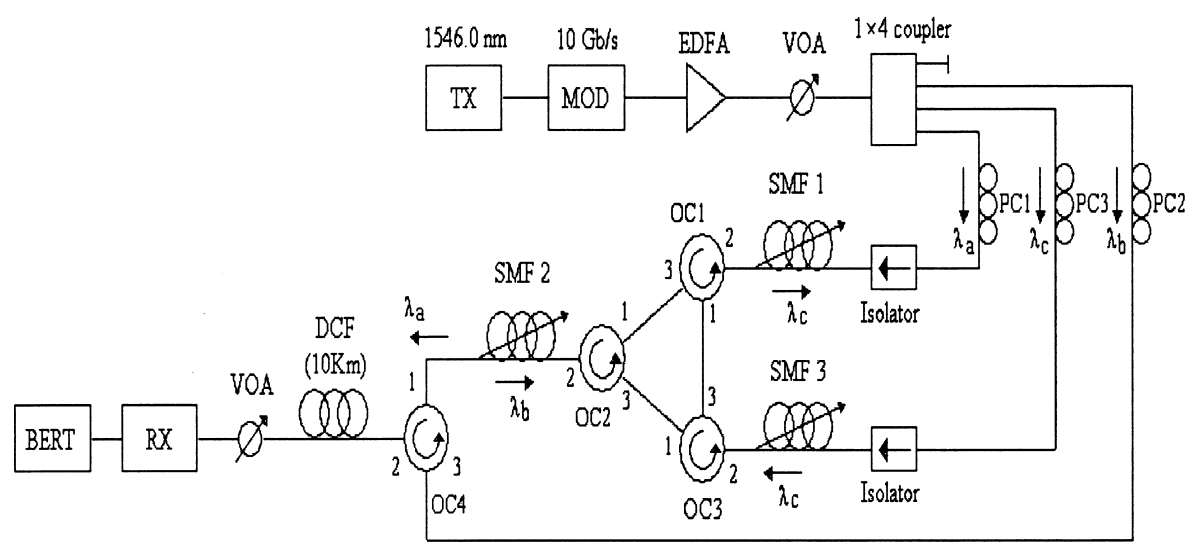

Figure 3. Experimental setup: EDFA: erbium-doped fiber amplifier, VOA: variable optical attenuator, OC: optical circulator, DCF: dispersion compensation fiber, BERT: bit error rate test set, PC: polarization controller.

adjust the appropriate launched power level. The modulated signal is then equally divided into four parts by using a $1 \times 4$ splitter. Except for one dummy port, the other three signals are imitated as three partly counter-propagating signals with $0 \mathrm{dBm}$ power level at points $\mathrm{a}, \mathrm{b}$, and c. Note that $\lambda_{a}, \lambda_{b}$, and $\lambda_{c}$ are the same wavelength of equal power. Three polarization controllers (PCs) are used to adjust the polarization state of signals.

The components that $\lambda_{a}$ pass through are single-mode fiber 1 (SMF1), optical circulator $1(\mathrm{OC} 1), \mathrm{OC} 2, \mathrm{SMF} 2, \mathrm{OC} 4$, dispersion compensating fiber (DCF), VOA, receiver (RX), then finally a bit error rate (BER) test set. The SMF1, SMF2, and SMF3 are three spools of SMF of $25 \mathrm{~km}$ equal length. The fiber attenuation of SMFs is $0.2 \mathrm{~dB} / \mathrm{km}$. The length between $\mathrm{OC} 1$ and $\mathrm{OC} 2$ is less than 2 meters so that the RB effect inside TMM is negligible here. In SMF1 there is $\lambda_{c}$, with which RB noise degrades the performance of $\lambda_{a}$. However, in SMF2, there is $\lambda_{c}$ with which RB noise degrades the performance of $\lambda_{a}$. The $10 \mathrm{DCF}$ is $10 \mathrm{~km}$ and can be used to compensate the pulse dispersion well. The receiver sensitivity is $-18.5 \mathrm{dBm}$ at a BER of $10^{-9}$. As for $\lambda_{a}$ transmission, the fiber interaction length is defined as the total distance of SMF1 + SMF2 that induces RB. The port-to-port isolation and insertion loss of the OCs are 50 and $0.6 \mathrm{~dB}$, respectively.

In this experiment we assume that the RB is the dominated reflection in the fiber. The power penalty is induced by homodyne crosstalk when two signals with identical wavelengths $\left(\lambda_{a}\right.$ and $\lambda_{b}, \lambda_{b}$ and $\lambda_{c}, \lambda_{b}$ and $\left.\lambda_{c}\right)$ and propagating in opposite direction with coherently mixed BER data are measured by varying the related polarization state of $\lambda_{a}, \lambda_{b}$, and $\lambda_{c}$. The total length of SMF for either signal $\lambda_{a}, \lambda_{b}$, or $\lambda_{c}$ is $50 \mathrm{~km}$ (i.e., $25+25 \mathrm{~km}$ ).

\section{Results and Discussion}

Figure 4 simulates the possible impact of RB signals $\lambda_{b}$, and $\lambda_{c}$ induces homodyne crosstalk to $\lambda_{a}$ under random polarization. As $\lambda_{a}$ travels from point a to TMM (i.e., in SMF1), the crosstalk level of RB signal $\left(\lambda_{c}\right)$ to $\lambda_{a}$ ranges from -60 to $-30 \mathrm{~dB}$. Such crosstalk has little impact on $\lambda_{a}$. However, as $\lambda_{a}$ travels from TMM to point $\mathrm{b}$ (i.e., in SMF2), the crosstalk level of RB signal $\left(\lambda_{b}\right)$ to $\lambda_{a}$ ranges from -45 to $-20 \mathrm{~dB}$. Such 


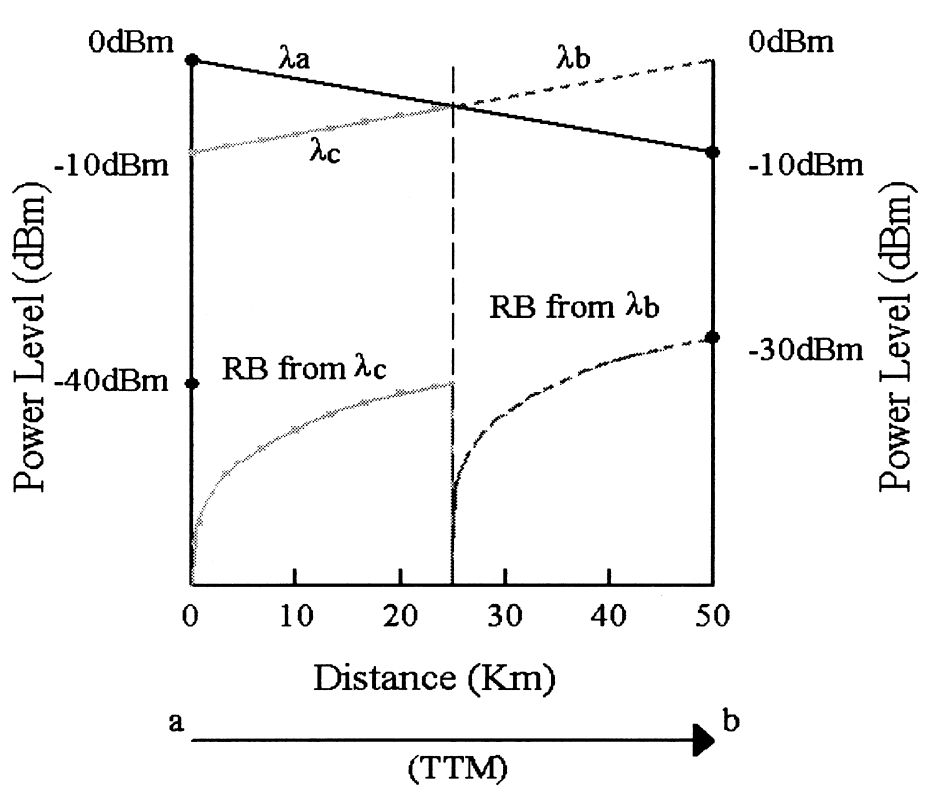

Figure 4. Simulation result of RB signals $\lambda_{c}$ inducing homodyne crosstalk to $\lambda_{a}$ in SMF1; and the RB signals $\lambda_{b}$ inducing homodyne crosstalk to $\lambda_{a}$ in SMF2.

crosstalk level may induce a $1.5 \mathrm{~dB}$ power penalty to $\lambda_{a}[7,9]$. Figure 5 shows the simulated result of power penalty $\lambda_{a}$ based on the best case of AC-coupled theory [9]:

$$
P=-10 \log \left[1-2 \times 10\left(\varepsilon_{\mathrm{dB}} / 20\right)\right](\text { AC coupled receiver }),
$$

where $\varepsilon_{\mathrm{dB}}$ is equivalent to $10 \log (\varepsilon)$ with $\varepsilon$ indicating the component crosstalk in decibels. When the fiber spacing $(L)$ is greater than $20 \mathrm{~km}, P$ can be expressed as

$$
P \sim-10 \log \left[1-2 S^{0.5}+S^{0.5} e^{-2 \alpha L}\right] .
$$

The $\mathrm{RB}$ noise here is random polarized.

Figure 6 shows the measured BER of $\lambda_{a}$ as a function of received optical power after it passed through SMF1 and SMF2. Compared to back-to-back measurement, the power penalty is $0.4,2.0$, and $4.0 \mathrm{~dB}$ for the best BER with PCs, random polarized without PCs (or shorten as normal PC), and the worst BER with PCs, respectively. Without PCs, the power penalty depends on the relative strength of Rayleigh backscattering and the signal. With PCs, the BER is best when two counter-propagated signals are in the orthogonal polarization state, whereas the worst BER occurs when two counter-propagation signals are in the parallel polarization states. In the latter case, the noise floor of BER is around $1 \times 10^{-9}$. We find that the measured power penalty $(2.0 \mathrm{~dB})$ in random polarization without PCs is a little bit larger than that of the theoretic prediction of $1.5 \mathrm{~dB}$. It may due to the extra reflection noises of fiber connection.

\section{Conclusion}

In a tri-directional wavelength-reused system, launching counter-propagating signals with orthogonal polarization-for example, using polarization controllers-can reduce 


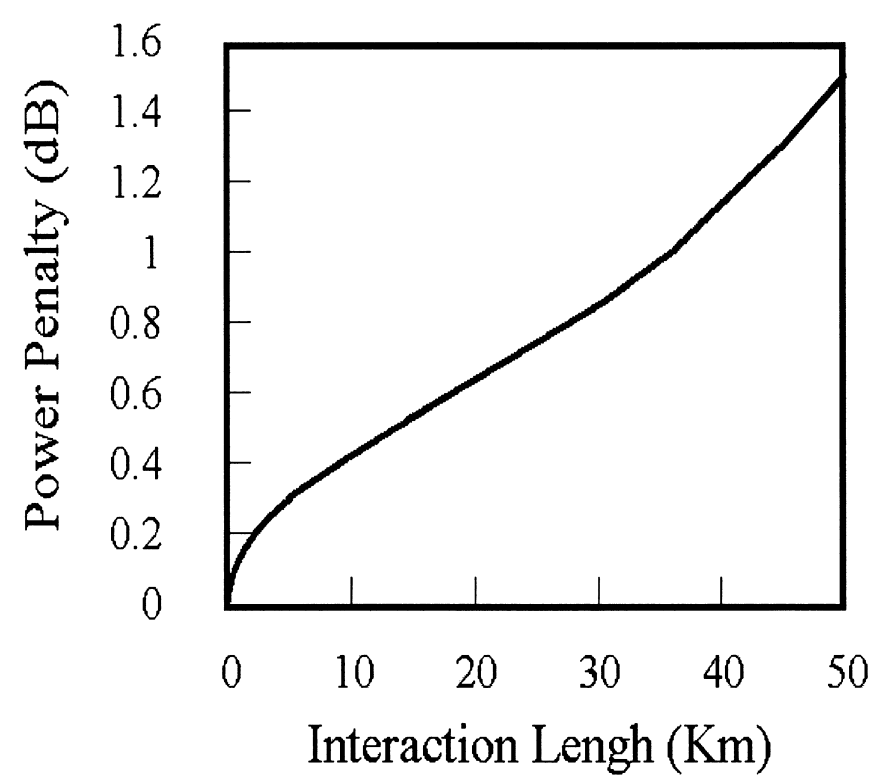

Figure 5. Simulation result of power penalty on $\lambda_{a}$ using the AC-coupler theory. The Rayleigh back signal is assumed to be random polarized.

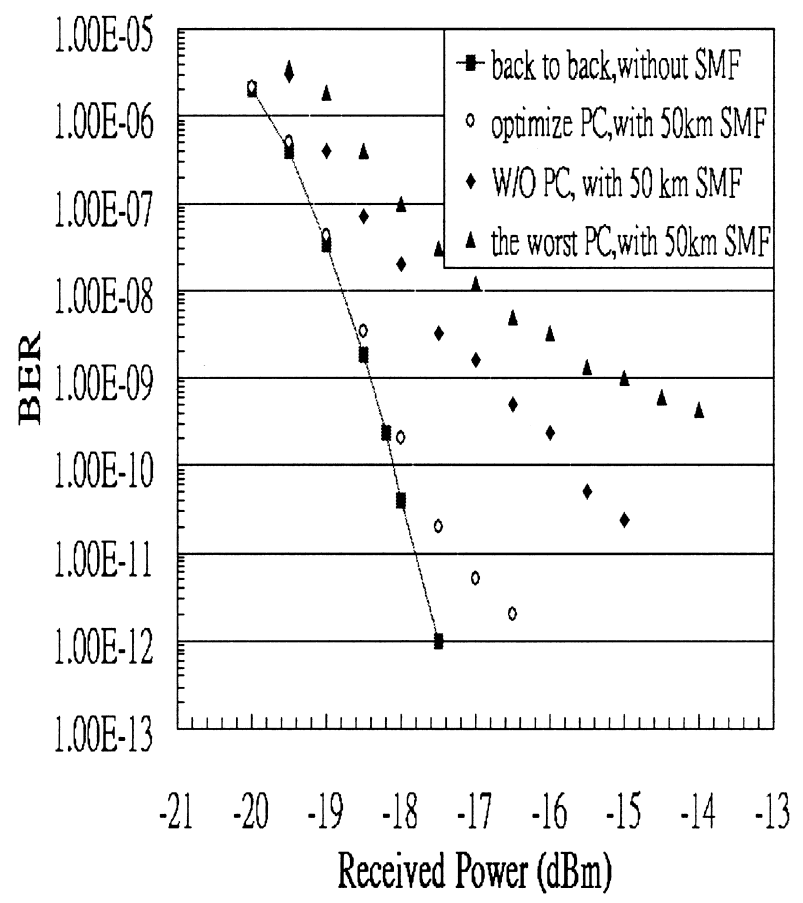

Figure 6. The measured BER results as a function of received optical power after $50(25+25) \mathrm{km}$ SMF with or without the polarization controllers. 
the impact of RB. With DCF for dispersion compensation and polarization controller, the result shows that the power penalty is reduced from the worst case of $4.0 \mathrm{~dB}$ to the best case of $0.4 \mathrm{~dB}$ for a $50 \mathrm{~km} \mathrm{SMF}$ in a $10.0 \mathrm{~Gb} / \mathrm{s}$ lightwave transmission system. The suggested interaction length of a tri-directional wavelength reused system is estimated to be over $50 \mathrm{~km}$ under a general $1.0 \mathrm{~dB}$ power penalty criteria. The proposed technique may find vast applications in WDM ring networks and tri-directional system where two or more signals add-drop and interchange, and cross-connect functions are necessary.

\section{References}

1. van Deventer, M. O., and O. J. Koning. 1995. Bidirectional transmission using an erbium-doped fiber amplifier without optical isolators. IEEE Photon. Technol. Lett. 7:1372-1374.

2. van Deventer, M. O. 1996. Fundamentals of bi-directional transmission over a single optical fiber. Boston: Kluwer Academic.

3. Wan, P., and J. Conradi. 1996. Impact of doubling Rayleigh backscattering noise on digital and analog fiber system. J. Lightwave Technol. 14:288-297.

4. Tsujikawa, K., K. Tajima, and M. Ohashi. 2000. Rayleigh scattering reduction method for silica-based optical fiber. J. Lightwave Technol. 18(11):1528-1532.

5. Takada, K. 2002. Improvement in signal-to-noise ratio of Rayleigh backscattering measurement using optical low coherence reflectometry. J. Lightwave Technol. 20:1001-1017.

6. Liaw, S. K., S.-L. Tzeng, and Y.-J. Hung. 2001. Rayleigh backscattering induced power penalty on bi-directional wavelength-reuse fiber systems. Opt. Commun. 188:63-67.

7. Ho, K.-P., C.-K. Chan, F. Tong, and L. K. Chen. 1998. Exact analysis of homodyne crosstalk induced penalty in WDM networks. IEEE Photon. Technol. Lett. 10:457-458.

8. Larsson, S. N. 1998. Improving Rayleigh-limited bidirectional optical transmission systems by using time-slotting and frequency detuning. 24th European Conference on Optical Communication, 1:485-486.

9. Goldstein, E. L., L. Eskildsen, and A. F. Elrefaie. 1994. Performance implications of component crosstalk in transparent lightwave networks. IEEE Photon. Technol. Lett. 6:657-659.

\section{Biographies}

Shien-Kuei Liaw received his B.S.E.E., M.S.E.E., and Ph.D. degrees from National Taiwan University, National Tsing-Hua University, and National Chiao-Tung University, Taiwan, in 1988, 1993, and 1999, respectively. He is currently an assistant professor in the Department of Electronics Engineering, National Taiwan University of Science and Technology. From 1993 to 1997, he was a research scientist with Chung-Hwa Telecommunication Laboratories, Taiwan. In 1996 he was a resident visitor at Bellcore, Red Bank, New Jersey. Dr. Liaw has published over 80 international journal articles and conference presentations. His research interests include optical fiber amplifiers, WDM lightwave subsystems, fiber Bragg grating devices, and fiber sensor technologies.

Yi-Fan Chiang is currently a student in the Department of Electronics Engineering, National Taiwan University of Science and Technology. He will be a graduate student at the National Tsing-Hwa University, Taiwan. In 1999 he received the second level of the Meter Electronic Certificate from the Department of CLA of the Executive, Yuan, Taiwan, R.O.C., and the diploma of the National Tai-Chung Industrial High School. His research interests include optical fiber amplifiers, fiber optic communication systems, and components.

Keang-Po Ho received his B.S. degree from National Taiwan University in 1991, and M.S. and Ph.D. degrees from the University of California at Berkeley in 1993 and 1995, 
respectively, all in electrical engineering. He is currently the Chief Technology Officer of StrataLight Communications, Campbell, California. He was a Research Scientist with Bellcore, currently Telcordia Technologies, Red Bank, New Jersey, from 1995 to 1997, and with the Department of Information Engineering of the Chinese University of Hong Kong between 1997 and 2001. He has published over 100 journal and conference articles and has several issued and pending patents. 